\title{
Harmonic Analysis of the DC Biased Epstein Frame-Like Core Model by the Harmonic Balance Finite Element Method
}

\author{
Xiaojun $\mathrm{Zhao}^{\# 1}$, Junwei $\mathrm{Lu}^{* 2}$, Lin $\mathrm{Li}^{\# 3}$, Zhiguang Cheng ${ }^{4}$ \\ ${ }^{\#}$ School of Electrical \& Electronic Engineering, North China Electric Power University \\ PO Box 10, 619 Yonghua North Street, Baoding, 071000, China \\ ${ }^{1} 158748295 @ 163 . c o m$ \\ ${ }^{3}$ lilin@ncepu.edu.cn \\ ${ }^{*}$ Griffith School of Engineering, Griffith University \\ Brisbane, 4111, Australia \\ ${ }^{2} j$.lu@griffith.edu.au \\ $R \& D$ Centre of Baoding Tianwei Group \\ Baoding, 071056, China \\ ${ }^{4}$ emlab@btwtc.com
}

\begin{abstract}
The DC biased problem of the Epstein Frame-like core model is investigated by using the Harmonic Balance Finite Element Method (HBFEM). It considers coupling between the electric circuit and the magnetic field. The magnetizing current and its harmonic components can be calculated directly in the harmonic domain. Comparing the calculated results with the experimental data shows consistency. The DC bias effect on the harmonic components of the exciting current is analyzed directly with the distribution of the magnetic flux visually observed through the harmonic solutions.
\end{abstract}

\section{INTRODUCTION}

With the development of HVDC transmission systems, researchers and engineers have paid more attention to the DC biased problem in HVDC transformers. The HVDC system operates in a monopolar mode and uses the earth as a return path. This will lead to a potential difference between the two converting plants. The resulting electric potential difference generates a direct current that flows into the windings of HVDC transformers through earthed neutrals in the AC network. Another form of DC bias, Geomagnetically Induced Current (GIC) [1], is caused by solar magnetic disturbances. The appearance of the DC in windings may damage the transformers and electric networks seriously.

Various methods have been used to investigate the DC biased phenomenon in HVDC transformers. The electric circuit model [2] and magnetic circuit model [3] were proposed by some reseachers to calculate the excitation current in winding, while the time-stepping finite element method [4] was used by many researchers to compute the magnetizing current and magnetic field. However, it is difficult to obtain accurate results from the electric and magnetic models (especially when magnetic field analysis is required to investigate the mechanism of the DC biased problem). The time-stepping finite element method is an effective method to calculate the transient magnetic field. However, accurate solutions of high order harmonics in the excitation current and flux density will reduce the effectiveness of time-stepping method.

In this paper the harmonic balance finite element method [5] (HBFEM) is introduced to solve the nonlinear magnetic field under DC bias conditions, considering coupling of electric circuits and magnetic fields. Due to the DC bias, zero order, odd order and even order harmonic components constitute the excitation current in transformer windings. All harmonic components of current and vector potential can be solved directly in the harmonic domain by the HBFEM approach. HBFEM can be an effective method of analysing the DC biased problem in transformers by comparing the calculated results with experimental data based on the Epstein Frame test.

\section{HARMONiC BALANCE Finite ELEMENT METHOD}

\section{A. Basic Formulation}

The nonlinear magnetic field can be presented by the following equation,

$$
\nabla \times \nu \nabla \times A+\sigma(\partial A / \partial t+\nabla \varphi)-J=0
$$

$\boldsymbol{A}$ is magnetic vector potential, $\boldsymbol{B}$ represents flux density, $\boldsymbol{v}$ and $\boldsymbol{\sigma}$ are reluctivity and conductivity respectively, $\boldsymbol{J}$ is exciting current.

For two-dimensional problems in nonlinear magnetic fields, equation (2) can be written as,

$$
\frac{\partial}{\partial x}\left(v \frac{\partial A}{\partial x}\right)+\frac{\partial}{\partial y}\left(v \frac{\partial A}{\partial y}\right)+\sigma\left(\frac{\partial A}{\partial t}+\frac{\partial \varphi}{\partial z}\right)=J
$$

It is assumed that the current flows in $\mathrm{Z}$ direction over an infinitely long distance. This means the electric scalar potential is constant, so the term including $\varphi$ can be neglected in (2). In light of Galerkin's method, the weighted residual is, 


$$
\begin{aligned}
G= & \iint_{\Omega_{e}}\left(\frac{\partial N_{i}^{e}}{\partial x} v \frac{\partial A}{\partial x}+\frac{\partial N_{i}^{e}}{\partial y} v \frac{\partial A}{\partial y}\right) d x d y+ \\
& \iint_{\Omega_{e}}\left(\sigma \frac{\partial A}{\partial t} N_{i}^{e}\right) d x d y-\iint_{\Omega_{e}}\left(J_{s} N_{i}^{e}\right) d x d y
\end{aligned}
$$

where $N_{i}$ is the interpolation function for a linear triangular element.

In the DC biased phenomenon we focus on the time-periodic solutions. This is actually a harmonic problem when alternating current and direct current are applied together. According to Fourier transformation theory all variables such as vector potential $\boldsymbol{A}$, flux density $\boldsymbol{B}$, excitation current $\boldsymbol{J}$ and reluctivity $\boldsymbol{v}$ can be approximated as a triangle series based on the harmonic balance method.

$$
\begin{aligned}
& A^{i}=A_{0}^{i}+\sum_{n=1}^{\infty}\left\{A_{n s}^{i} \sin (n \omega t)+A_{n c}^{i} \cos (n \omega t)\right\} \\
& J_{s}=J_{0}+\sum_{n=1}^{\infty}\left\{J_{n s} \sin (n \omega t)+J_{n c} \cos (n \omega t)\right\} \\
& B_{x}^{e}=B_{x 0}^{e}+\sum_{n=1}^{\infty}\left\{B_{x n s}^{e} \sin (n \omega t)+B_{x n c}^{e} \cos (n \omega t)\right\} \\
& B_{y}^{e}=B_{y 0}^{e}+\sum_{n=1}^{\infty}\left\{B_{y n s}^{e} \sin (n \omega t)+B_{y n c}^{e} \cos (n \omega t)\right\} \\
& v=v_{0}+\sum_{n=1}^{\infty}\left\{v_{n s} \sin (n \omega t)+v_{n c} \cos (n \omega t)\right\}
\end{aligned}
$$

Considering the orthogonal characteristics of trigonometric functions, the HBFEM matrix equation for a single element can be obtained from (3) and (4),

$$
\begin{aligned}
G^{e}= & {\left[S^{e}\right]\left\{A^{e}\right\}+\left[N^{e}\right]\left\{A^{e}\right\}-\left\{K^{e}\right\} } \\
= & \frac{1}{4 \Delta^{e}}\left[\begin{array}{ccc}
\left(b_{1} b_{1}+c_{1} c_{1}\right) D & \left(b_{1} b_{2}+c_{1} c_{2}\right) D & \left(b_{1} b_{3}+c_{1} c_{3}\right) D \\
\left(b_{2} b_{1}+c_{2} c_{1}\right) D & \left(b_{2} b_{2}+c_{2} c_{2}\right) D & \left(b_{2} b_{3}+c_{2} c_{3}\right) D \\
\left(b_{3} b_{1}+c_{3} c_{1}\right) D & \left(b_{3} b_{2}+c_{3} c_{2}\right) D & \left(b_{3} b_{3}+c_{3} c_{3}\right) D
\end{array}\right] \\
& \times\left\{\begin{array}{l}
A_{1}^{e} \\
A_{2}^{e} \\
A_{3}^{e}
\end{array}\right\}+\frac{\sigma \omega \Delta^{e}}{12}\left[\begin{array}{ccc}
2 N & N & N \\
N & 2 N & N \\
N & N & 2 N
\end{array}\right]\left\{\begin{array}{l}
A_{1}^{e} \\
A_{2}^{e} \\
A_{3}^{e}
\end{array}\right\}-\left\{\begin{array}{l}
K_{1}^{e} \\
K_{2}^{e} \\
K_{3}^{e}
\end{array}\right\} \\
= & 0
\end{aligned}
$$

in which $b_{i e}=y_{j e}-y_{k e}, c_{i e}=x_{k e}-x_{j e}, D$ is the reluctivity matrix and $N$ is the harmonic matrix,

$$
D^{e}=\left[\begin{array}{llllll}
d_{11} & d_{12} & d_{13} & d_{14} & d_{15} & \cdots \\
d_{21} & d_{22} & d_{23} & d_{24} & d_{25} & \cdots \\
d_{31} & d_{32} & d_{33} & d_{34} & d_{35} & \cdots \\
d_{41} & d_{42} & d_{43} & d_{44} & d_{45} & \cdots \\
d_{51} & d_{52} & d_{53} & d_{54} & d_{55} & \cdots \\
\cdots & \cdots & \cdots & \cdots & \cdots & \ddots
\end{array}\right]
$$

$$
=\frac{1}{2}\left[\begin{array}{cccccc}
2 v_{0} & v_{1 s} & v_{1 c} & v_{2 s} & v_{2 c} & \cdots \\
2 v_{1 s} & 2 v_{0}-v_{2 c} & v_{2 s} & v_{1 c}-v_{3 c} & -v_{1 s}+v_{3 s} & \cdots \\
2 v_{1 c} & & 2 v_{0}+v_{2 c} & v_{1 s}+v_{3 s} & v_{1 c}+v_{3 c} & \cdots \\
2 v_{2 s} & & & 2 v_{0}-v_{4 c} & v_{4 s} & \cdots \\
2 v_{2 c} & & \text { Symmetry } & & 2 v_{0}+v_{4 c} & \cdots \\
\cdots & & & & & \ddots
\end{array}\right]
$$$$
N^{e}=\left[\begin{array}{cccccc}
0 & 0 & 0 & 0 & 0 & \cdots \\
0 & 0 & -1 & 0 & 0 & \cdots \\
0 & 1 & 0 & 0 & 0 & \cdots \\
0 & 0 & 0 & 0 & -2 & \cdots \\
0 & 0 & 0 & 2 & 0 & \cdots \\
\cdots & \cdots & \cdots & \cdots & \cdots & \ddots
\end{array}\right]
$$

B. Coupling between electric circuits and the magnetic field

When electrical devices are excited by voltage, such as transformers under DC bias, the excitation current is unknown and equation (1) is no longer applicable. In this case the coupling between the electric circuit and the magnetic field should be taken into account [6].

According to Kirchhoff's law the applied voltage on the external port can be defined as follows,

$$
\begin{aligned}
V_{\text {ink }} & =V_{k}+R_{k} I_{k}+L_{k} \frac{d I_{k}}{d t}+\frac{1}{C_{k}} \int I_{k} d t \\
& =\left[C_{k}^{e}\right]\{A\}+S_{c k}\left[Z_{k}\right]\left\{J_{k}\right\}
\end{aligned}
$$

$V_{\text {ink }}$ is the input voltage of circuit $k, V_{k}$ is the corresponding induced electromotive force, $S_{c k}$ and $Z_{k}$ are the cross areas and impedance of winding $k$ respectively, $C_{k}$ and $L_{k}$ are the capacitance and inductance respectively in circuit $k$.

The induced electromotive force can be gained based on Faraday's law,

$$
\begin{aligned}
V_{k} & =\frac{d \Psi}{d t}=\frac{d}{d t} \iint \vec{B} \cdot d \vec{S} \\
& =\frac{d}{d t} \iint \nabla \times \vec{A} d \vec{S}=\frac{d}{d t} \oint_{f} \vec{A} d \vec{l} \\
& =\frac{\omega N_{k} d_{0}}{3 S_{c k}}\left[\begin{array}{lll}
N & N & N
\end{array}\right]\left\{\begin{array}{l}
\left\{A^{1}\right\} \\
\left\{A^{2}\right\} \\
\left\{A^{3}\right\}
\end{array}\right\}
\end{aligned}
$$

The expression of the impedance matrix is given by (10) and (11) respectively,

$\left[Z_{k}\right]=\left[\begin{array}{cccc}Z_{0 k} & 0 & 0 & \ldots \\ 0 & Z_{1 k} & 0 & \ldots \\ 0 & 0 & Z_{2 k} & \cdots \\ \vdots & \vdots & \vdots & \ddots\end{array}\right]$ 


$$
\begin{aligned}
Z_{n k} & =Z_{R n k}+Z_{C n k}+Z_{L n k} \\
& =\left[\begin{array}{cc}
R+n \omega L-\frac{1}{n \omega C} & 0 \\
0 & R+n \omega L-\frac{1}{n \omega C}
\end{array}\right]
\end{aligned}
$$

The system matrix equation (1) can be rewritten if the right term representing the excitation characteristic is moved to the left hand side,

$$
[S]\{A\}+[M]\{A\}-\left[G_{k}\right]\left\{J_{k}\right\}=0
$$

The new system matrix equation considering the applied voltage can be obtained by combining (7) with (11),

$$
\left[\begin{array}{cccccc}
{[H]} & -\left[G_{1}\right] & -\left[G_{2}\right] & \cdots & -\left[G_{k}\right] & \cdots \\
{\left[C_{1}\right]} & S_{c 1}\left[Z_{1}\right] & 0 & 0 & 0 & \cdots \\
{\left[C_{2}\right]} & 0 & S_{c 2}\left[Z_{2}\right] & 0 & 0 & \cdots \\
\vdots & 0 & 0 & \ddots & 0 & \cdots \\
{\left[C_{k}\right]} & 0 & 0 & 0 & S_{c k}\left[Z_{k}\right] & \cdots \\
\vdots & \vdots & \vdots & \vdots & \vdots & \ddots
\end{array}\right]\left\{\begin{array}{c}
\{A\} \\
\left\{J_{1}\right\} \\
\left.J_{2}\right\} \\
\vdots \\
\left\{J_{k}\right\} \\
\vdots
\end{array}\right\}=\left\{\begin{array}{c}
0 \\
\left\{V_{i n 1}\right\} \\
\left.V_{i n 2}\right\} \\
\vdots \\
\left\{V_{i n k}\right\} \\
\vdots
\end{array}\right\}
$$

The vector potentials and current densities can be solved simultaneously by the above equation.

\section{CALCULATION RESUltS AND FIELD ANALYSIS}

An Epstein Frame-like core model made by the TianWei Group, Baoding, has been tested under different DC bias conditions. The excitation current without a DC bias is selected as the benchmark, which makes the flux density in silicon steel reach rated value in operation of transformers. The peak value of the benchmark current $I_{0}$ is 1.68 ampere.

\section{A. Excitation Current}

The DC bias is applied in incremental proportions of the benchmark current: $25 \%, 50 \%, 75 \%$ and $100 \%$. Figures $1-4$ compare calculated values with experimental data.

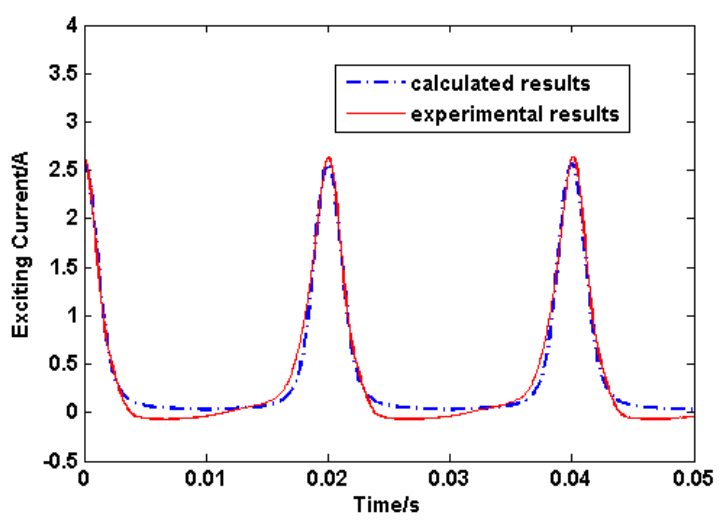

Fig.1 Excitation current under 25\% DC bias

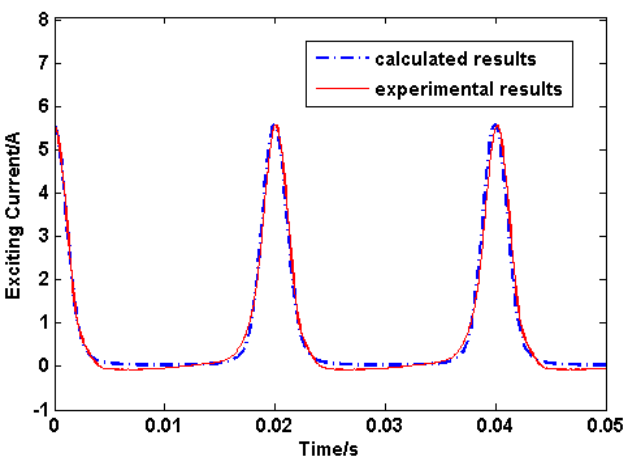

Fig.2 Excitation current under 50\% DC bias

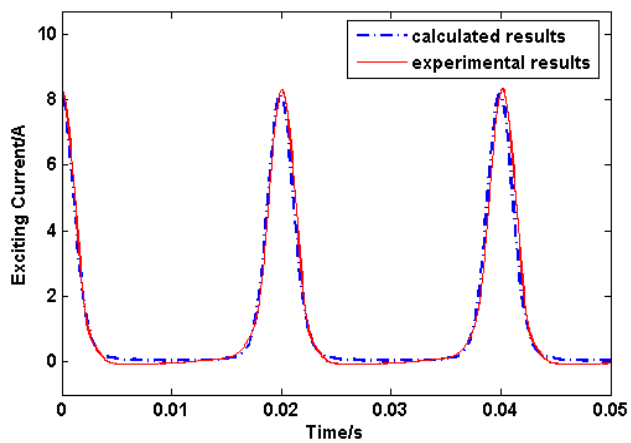

Fig.3 Excitation current under 75\% DC bias

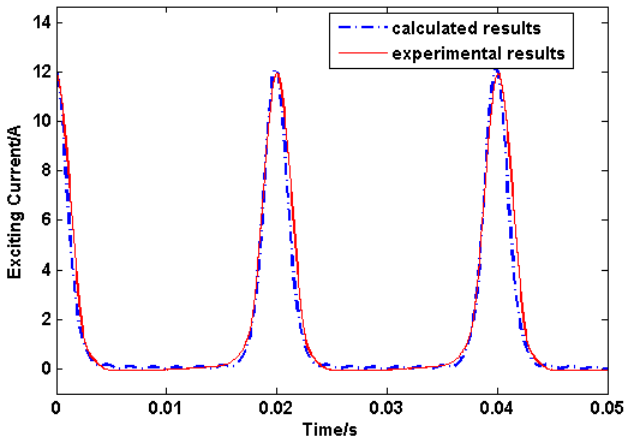

Fig.4 Excitation current under 100\% DC bias

\section{B. Harmonic Analysis}

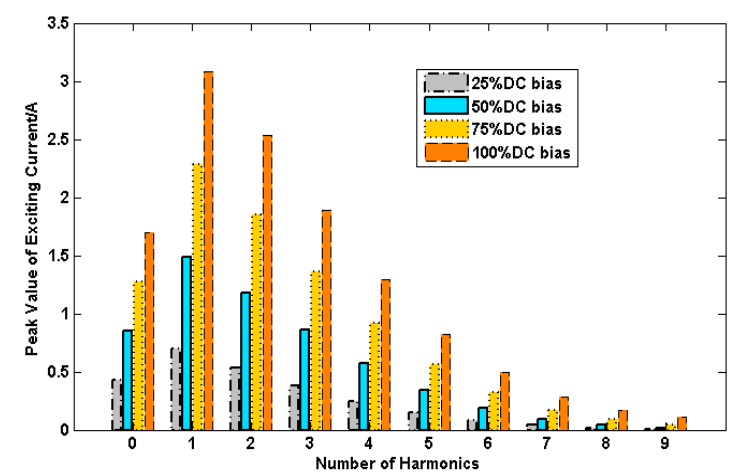

Fig. 5 Peak value of excitation current against harmonic components under different DC biases 
Unlike the Fourier transforming process in the time-stepping solution, all harmonic components in the magnetizing current can be obtained directly from the solved current density. The histogram in Fig.5 shows the contribution of different harmonic components in the excitation current under different DC biases. There is consistency in the computation and measurement results obtained from the magnetizing current waveforms. This proves the effectiveness of the harmonic balance finite element method in solving the DC biased problem in transformers.

The excitation current includes odd harmonics when the transformer operates without DC bias. However, even components will appear because the direct current invades the transformer windings. The DC bias results in serious magnetic saturation and half-cycle saturation of the magnetizing current. Therefore, the relationship between DC bias and other harmonic components should be considered by using harmonic analysis.

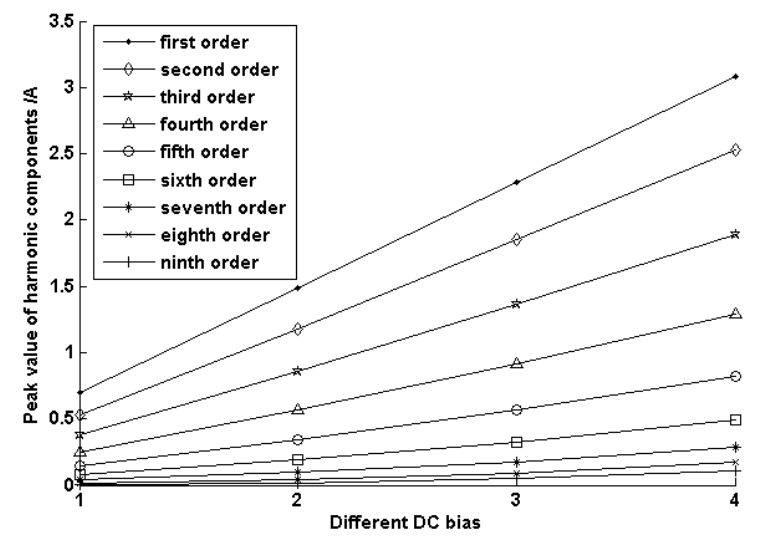

Fig.6 Peak value of harmonic components under different DC biases

Fig.5 shows that while the size of all harmonic components increases when additional DC bias is applied, the growth rate varies in different components. The growth tendency of each harmonic is shown in Fig.6. The numbers $1,2,3,4$ in the horizontal coordinate represent different proportions $(25 \%, 50 \%, 75 \%, 100 \%)$ of the DC bias benchmark respectively. It is obvious that the fundamental and second harmonic components increase near-linearly, while higher order harmonics (such as the third and fourth) grow faster rather than linearly. Higher order components grow faster than lower order components.

\section{Flux Distribution}

The magnetic induction in each element is represented in the form of a harmonic component in equation (1). It can be calculated directly from the field equation (12), so the flux distribution in the steel region can be given directly from the solution.

Fig.7 (a) through (d) show the flux distribution of the harmonic components in the magnetic core. There are significant differences between DC and AC components in terms of flux distribution.

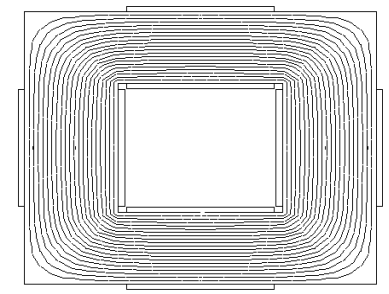

a. DC component

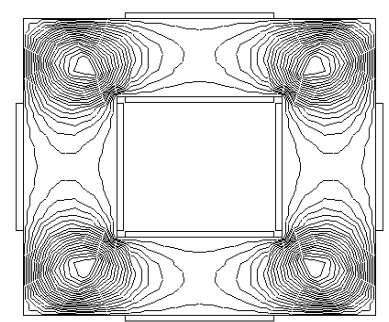

c. Second harmonic component

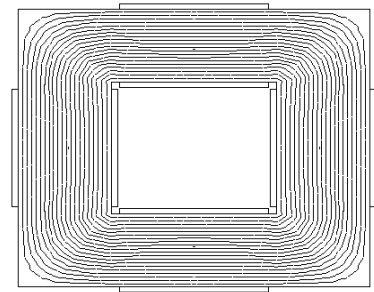

b. Fundamental component

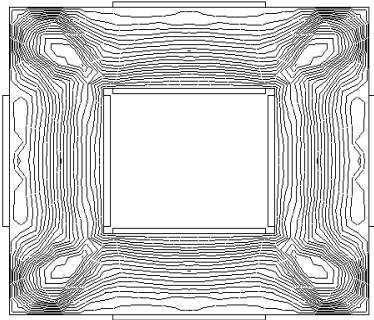

d. Third harmonic component
Fig.7 Magnetic flux distribution of DC and AC components

\section{CONCLUSIONS}

The HBFEM has been introduced to solve the DC biased problems in transformers. It is proven to be an effective and efficient approach to the analysis of steady-state nonlinear magnetic fields in the harmonic domain. Harmonic analysis of magnetizing current and magnetic field is presented. The effectiveness of the HBFEM for the DC biased problem is indicated in the consistency between the calculated and experimental results.

\section{ACKNOWLEDGEMENT}

This work was supported by National Natural Science Foundation of China under Grant No.50677016 and the Australian Research Council (ARC) Foundation under Grant No.DP0772205. The DC biased test was carried out in the EMLAB of R\&D Centre, Baoding Tianwei Group Co., LTD. The authors thank the anonymous reviewers for their comments that improve the presentation in this paper.

\section{REFERENCES}

[1] O. Biro, S. Ausserhofer, G. Buchgraber, K.Preis and W. Seitlinger, "Prediction of magnetizing current waveform in a single-phase power transformer under DC bias," Science, Measurement \& Technology, IET, vol. 1, pp. 2-5, Jan. 2007.

[2] Xiaoping Li, Xishan Wen, Cixuan Cheng, "Simulating analysis of excitation current of single phase transformer on DC bias", High Voltage Engineering, vol. 31, pp. 8-10, Sep. 2007.

[3] Lin Cao, Jie Zhao, and Jinliang He, "Improved power transformer model for DC biasing analysis considering transient leakage reluctance," in Proc. ICPST, 2006, p. 1.

[4] Yingying Yao, "Research on the DC bias phenomena of large power transformers," D. Eng. thesis, Shenyang University of Technology, Shenyang, China, Nov. 2000.

[5] S. Yamada, K. Bessho, "Harmonic field calculation by the combination of finite element analysis and harmonic balance method," IEEE Transactions on Magnetics, vol. 24, pp. 2588-2590, Nov. 1988.

[6] Junwei Lu, S. Yamada, K.Bessho, "Harmonic balance finite element method taking account of external circuit and motion," IEEE Transactions on Magnetics, vol. 27, pp. 4024-4027, Nov. 1991. 